\title{
An Investigation into the Relationship between N-Terminal Pro B-Type Natriuretic Peptide (NT-proBNP) and Fragmented QRS in Patients with Chronic Heart Failure
}

\author{
Javad Ramezani ${ }^{1}$, Zahra Sharifi' ${ }^{2}$, Shirin Sadat Ghiasi ${ }^{3}$, Majid Jalalyazdi ${ }^{4}$
}

\begin{abstract}
${ }^{1}$ Assistant Professor, Cardiologist, Department of Cardiology, Faculty of Medicine, Mashhad University of Medical Sciences, Mashhad, Iran. ${ }^{2}$ Resident, MD, Department of Cardiology, Faculty of Medicine, Mashhad University of Medical Sciences, Mashhad, Iran. ${ }^{3}$ Medical Student, MD, Researcher, Department of Cardiology, Faculty of Medicine, Mashhad University of Medical Sciences, Mashhad, Iran. ${ }^{4}$ Assistant Professor, Cardiologist, Department of Cardiology, Faculty of Medicine, Mashhad University of Medical Sciences, Mashhad, Iran.
\end{abstract}

\section{ABSTRACT}

\section{BACKGROUND}

Heart failure is a clinical syndrome characterized by a constellation of symptoms and signs often caused by a structural and/or functional cardiac abnormality resulting in reduced cardiac output and/or elevated intracardiac pressures. Fragmented QRS (fQRS) complex is associated with fibrosis or myocardial scar in patients with structural heart disease. Patients, especially cardiologists, are particularly concerned about heart failure in people suffering from dyspnoea. The prevalence of this disease is rising in industrial countries. The relationship between NT-proBNP and fragmented QRS in patients with chronic heart failure was investigated in this study.

\section{METHODS}

The population included 42 patients suffering from chronic heart failure. fQRS was described as $\geq 1$ additional deflection, including the peak of the R-wave or the nadir of S-wave, in at least two continuous leads. According to the absence of fQRS [fQRS $(-)]$ or presence of fQRS ( $n=22$ and $n=20$, respectively), the patients were assigned to two groups. The statistical package for social sciences (SPSS, version 22) was used to analyse the data. Furthermore, the normality of the data was investigated by the Shapiro-Wilk test. The chi-square test and independent t-test were used to compare the variables.

\section{RESULTS}

42 patients (18 women and 24 men) aged $54.78 \pm 6.43$ years participated in this study. Given the fQRS at hospitalization, the patients were assigned to fQRS (-) (n= $20)$ and fQRS $(+)(n=22)$ groups. The results showed that NT-proBNP level was significantly lower in fQRS $(-)$ group $(\mathrm{p}<0.001)$, compared to fQRS $(+)$ group. There was a significant difference between the two groups in terms of NYHA, blood pressure, hypertension, diabetes and hyperlipidaemia. The groups were compared in terms of demographic characteristics, angiography including hyperlipidaemia, and history of cardiovascular diseases. The fQRS (+) group exhibited a higher proportion of hyperlipidaemia (63.40\%), troponin I $(34.33 \pm 21.87)$ and NT-proBNP $(242.34 \pm 42.65)$ than the fQRS $(-)$ group.

\section{CONCLUSIONS}

The results showed a significant relationship between fQRS and the higher levels of NT-proBNP. The fQRS was associated with increased NT-proBNP and pronounced $\mathrm{LV}$ end-diastolic pressure in heart failure patients.

\section{KEY WORDS}

Heart Failure, NT-proBNP, Fragmented QRS
Corresponding Author: Majid Jalalyazdi

Department of Cardiology,

School of Medicine,

Mashhad University of Medical Sciences,

Mashhad, Iran.

E-mail:drmajidjalayazdi@yahoo.com

DOI: $10.14260 /$ jemds/2019/578

Financial or Other Competing Interests: None.

How to Cite This Article:

Ramezani J, Sharifi Z, Ghiasi SS, et al. An investigation into the relationship between $N$-terminal pro B-type natriuretic peptide (NT-proBNP) and fragmented QRS in patients with chronic heart failure. J. Evolution Med. Dent. Sci. 2019;8(34): 2658-2661, DOI 10.14260/jemds/2019/578

Submission 07-04-2019, Peer Review 12-08-2019, Acceptance 19-08-2019, Published 26-08-2019. 


\section{BACKGROUND}

Heart failure presents a multifactorial, systemic disease, in which, after a cardiac injury, structural, neurohumoral, cellular, and molecular mechanisms are activated and act as a network to maintain physiological functioning.(1) Also, this is a clinical syndrome characterized by a constellation of symptoms (Dyspnoea, orthopnoea, lower limb swelling) and signs (Elevated jugular venous pressure, pulmonary congestion) often caused by a structural and/or functional cardiac abnormality resulting in reduced cardiac output and/or elevated intracardiac pressures. ${ }^{2}$ The incidence and prevalence of heart failure increase strikingly with age and make heart failure the most common reason for hospitalization among older adults. Although outcomes for older adults with heart failure have improved over time, mortality, hospitalization, and rehospitalization rates remain high.(3) Patients, especially cardiologists, are particularly concerned about heart failure in people suffering from dyspnoea. The prevalence of this disease is rising in the industrial countries. It reportedly accounts for 600.000 cases annually.(4) Heart failure can also be regarded as an economic issue which imposes a great deal of costs on the medical systems and leads to disability and decreased ability to work.(5) Given the economic costs imposed on society due to the prevalence of heart failure as well as the fact that this disease affects the quality of life of the patient, rapid diagnosis and immediate treatment of patients with heart failure is required to prevent or delay the progression of the disease.(6) Although echocardiography is the gold standard for diagnostic purposes, it is usually not available in many primary care settings, it is expensive and may not be accurate in many acute conditions.(7) Furthermore, echocardiography is time-consuming and requires the individual's skill and expertise to be performed and interpreted. If a patient with dyspnoea suffers from heart failure and it remains undiagnosed, the disease may progress and kill the patient finally. ${ }^{(8)}$

Fragmented QRS (fQRS) complex is associated with fibrosis or myocardial scar in patients with structural heart disease is recorded on the surface of electrocardiograms (ECGs).(9) According to the previous studies, fQRS complex was a good predictor of adverse cardiovascular conditions such as cardiac death. Meanwhile, some other studies found that myocardial scar or conduction disturbance may cause significant abnormality with the fQRS complex. For example, patients with left ventricular hypertrophy who also suffer from a notch in the fQRS complex may show a defects in the intraventricular conduction.(10) The injured tissues around an infarct scar may lead to RSR' pattern in the QRS.(11) Despite the fact the past research has focused on the relationship between cardiac complications and fQRS, there is still little information about the prognostic and diagnostic values of the QRS complex abnormalities. According to some studies, fQRS was associated with arrhythmic events and mortality in patients with structural heart disease (SHD). According to some studies, patients suffering from coronary artery disease who had fQRS showed myocardial conduction block. This may be attributed to the myocardial scar. To detect this complication, myocardial single photon emission tomography was used. Using fQRS in patients with prior myocardial infarction could contribute to the evaluation of CAD. Further showed that $\mathrm{fQRS}$ can be used as a potential indicator to diagnose or predict the ischemic cardiomyopathy and some electrical diseases. ${ }^{(12)}$ We aimed to examine the relationship between fragmented QRS and NT-proBNP in patients with chronic heart failure.

\section{METHODS}

This diagnostic clinical trial study was conducted on patients referring to the clinic from March to June 2017. The patients provided informed consent to work on the plan. According to the criteria of the European Society of Heart Association, the inclusion criteria were the approval of acute myocardial infarction (AMI) and patients whose main complaint was dyspnoea. Patients with heart failure who received guideline mediated treatment were included in this study. According to the inclusion and exclusion criteria, 42 patients participated in the study. Several morphologies of the QRS $(<120 \mathrm{~ms})$ are included in the fQRS, including notching in the nadir of the $\mathrm{S}$ wave and additional R wave (R'). Given the fQRS [fQRS (-)] or fQRS [fQRS $(+)](n=22$ and $n=20$, respectively), the patients were assigned to two groups. All the patients were asked to give their blood sample within 24 hours. Accordingly, Creatinine, NT-proBNP troponin I and urea were measured on a daily basis.

\section{Statistical Analysis}

The statistical package for social sciences (SPSS, version 22) was used to analyse the data. Furthermore, the normality of the data was investigated by the Shapiro-Wilk test. The chisquare test and independent t-test were used to compare the variables.

\section{RESULTS}

42 patients (18 women and 24 men) aged $54.78 \pm 6.43$ years participated in this study. Given the fQRS at hospitalization, the patients were assigned to fQRS $(-)(n=20)$ and fQRS $(+)$ $(n=22)$ groups. Table 1 shows the echocardiographic and baseline demographic characteristics of the patients. There was a significant difference between the two groups in terms of NYHA, blood pressure, hypertension, diabetes and hyperlipidaemia. Table 2 shows the laboratory characteristics of the fQRS groups.

\begin{tabular}{|c|c|c|c|}
\hline & $\begin{array}{c}\text { fQRS (+) Group } \\
(\mathbf{n = 2 2})\end{array}$ & $\begin{array}{c}\text { fQRS (-) Group } \\
(\mathbf{n = 2 0 )}\end{array}$ & p Value \\
\hline Age, years & $55.45 \pm 6.57$ & $53.56 \pm 7.45$ & 0.343 \\
\hline Men, n (\%) & $13(63.40)$ & $11(50.00)$ & 0.654 \\
\hline Current smoker, n (\%) & $12(54.54)$ & $11(55.00)$ & 0.347 \\
\hline Hypertension, n (\%) & $11(50.00)$ & $9(45.00)$ & 0.784 \\
\hline Hyperlipidemia, n (\%) & $13(63.40)$ & $12(60.00)$ & 0.459 \\
\hline Diabetes, n (\%) & $8(36.36)$ & $8(40.00)$ & 0.454 \\
\hline SBP, mmHg & $117 \pm 32$ & $117 \pm 21$ & 0.132 \\
\hline DBP, mmHg & $70 \pm 13$ & $70 \pm 14$ & 0.787 \\
\hline NYHA class, n (\%) & & & 0.346 \\
\hline II & $10(45.56)$ & $9(45.00)$ & 0.436 \\
\hline III & $12(54.54)$ & $11(55.00)$ & 0.236 \\
\hline Ejection Fraction & $39 \pm 6$ & $40 \pm 5$ & \\
\hline \multicolumn{2}{|c|}{ Table 1. Baseline Demographic and Echocardiographic } \\
Characteristics of fQRS (+) and fQRS (-) Groups \\
\hline \multicolumn{4}{|l}{} \\
\hline
\end{tabular}




\begin{tabular}{|c|c|c|c|}
\hline & $\begin{array}{c}\text { fQRS (+) Group } \\
(\mathbf{n = 2 2})\end{array}$ & $\begin{array}{c}\text { fQRS (-) Group } \\
(\mathbf{n = 2 0 )}\end{array}$ & p Value \\
\hline Hemoglobin, g/dL & $13.2 \pm 2.3$ & $13.5 \pm 1.99$ & 0.143 \\
\hline Glucose, $\mathrm{mg} / \mathrm{dL}$ & $90 \pm 11$ & $92 \pm 8$ & 0.425 \\
\hline Creatinine, $\mathrm{mg} / \mathrm{dL}$ & $1.08 \pm 9.8$ & $1.10 \pm 0.14$ & 0.656 \\
\hline Troponin I, $\mu \mathrm{g} / \mathrm{L}$ & $34.33 \pm 21.87$ & $22.56 \pm 12.7$ & 0.002 \\
\hline Total cholesterol, mg/L & $210.43 \pm 21.54$ & $215.67 \pm 34.21$ & 0.433 \\
\hline LDL-cholesterol, mg/L & $100.56 \pm 10.87$ & $104.76 \pm 10.74$ & 0.675 \\
\hline HDL-cholesterol, mg/L & $44.54 \pm 6.75$ & $42.54 \pm 6.36$ & 0.187 \\
\hline Triglyceride, mg/L & $160.65 \pm 45.21$ & $157.87 \pm 33.17$ & 0.823 \\
\hline NT-proBNP, pg/mL & $242.34 \pm 42.65$ & $117.76 \pm 65.76$ & 0.001 \\
\hline \multicolumn{3}{|c|}{ Table 2. Baseline Laboratory Characteristics of fQRS (+) } \\
and fQRS (-) Groups \\
\hline \multicolumn{4}{|c|}{}
\end{tabular}

There was no significant difference between the two groups in terms of NYHA, hypertension, hypertension, diabetes. However, according to Table 2, there is a significant difference between the two fQRS (+) and fQRS $(-)$ groups in terms of troponin I and NT-proBNP at level 95\% ( $\mathrm{p}$ value= 0.002 and $p$ value $=0.001$, respectively). However, no significant difference was found between the two groups in terms of other laboratory characteristics. The groups were compared in terms of demographic characteristics, angiography including hyperlipidaemia, and history of cardiovascular diseases. The fQRS $(+)$ group exhibited a higher proportion of hyperlipidaemia $(63.40 \%)$, troponin I $(34.33 \pm 21.87)$ and NT-proBNP $(242.34 \pm 42.65)$ than the fQRS (-) group.

\section{DISCUSSION}

Heart failure (HF) is the leading cause of morbidity and mortality worldwide and negatively impacts quality of life, healthcare costs, and longevity (13). In this research, we investigated the relationship between fragmented QRS and NT-proBNP in patients with chronic heart failure. According to the findings, NT-proBNP level was significantly lower in fQRS (-) group ( $\mathrm{p}<0.001)$, compared to fQRS $(+)$ group. The natriuretic peptides are not highly recommended for diagnosing heart failure. However, they can estimate the prognosis and severity of the heart failure. NT-proBNP and type natriuretic peptide (BNP) are two of the most widely used natriuretic peptides, which are secreted in response to stretch.(14) These biomarkers can help detect natriuretic peptides in blood.(14) As NYHA functional class worsens in terms of function, the amount of natriuretic peptides significantly. Meanwhile, the result showed that the amount of natriuretic peptide was higher in HFrEF than HFpEF. This difference can help increase the concentrations of natriuretic peptides. According to the results, patients with acute HF had higher BNP and NT-proBNP, compared to stable patients with CHF. However, further research is required to confirm this finding. It is necessary to specify the amount of natriuretic peptide in stable patients in order to provide an insight into the changes in HF symptoms.(15,16) Zhang et al. (2017) examined the prognostic value of the complex QRS (fQRS) for microvascular reperfusion and changes in left ventricular function in patients with myocardial infarction. Zhang et al. (2017) examined 216 patients. The patients were divided into two groups (of 126 and 90) based on the presence or absence of fQRS. In both groups, troponin levels, NT-proBNP and creatine kinase were studied. Zhang stated that in the analysis of logistic regression, LV-FV (LVEF) NT-proBNP,
Troponin I and microvascular reperfusion were associated with fQRS. Moreover, Zhang stated that the presence of fQRS was not only significantly associated with the myocardial microvascular reperfusion and left ventricular function, but also with the prognosis of STEMI. However, our study showed that fQRS was directly associated with NTproBNP.(16)

Barman and et al. (2019) aimed to investigate the relationship between the presence of fragmented QRS (fQRS) on electrocardiogram (ECG) and plasma galectin-3 levels in patients with heart failure (HF) and severely decreased left ventricular ejection fraction. They show fQRS and serum galectin-3 levels are associated with myocardial fibrosis and are associated with poor prognosis in heart failure. In their study, a positive correlation was found between serum galectin-3 levels and fQRS on ECG.(17) Zhao et al (2018) showed that there was an association between fragmented QRS and NT-proBNP among patients with myocardial infarction. In this study, 216 patients were studied. Zhao stated that the presence of fQRS was significantly associated with NT-proBNP and left ventricular ejection fraction, which can predict left ventricular function in patients with STEMI. fQRS is a prognostic marker of ventricular cytology dysfunction. This was in good agreement with the results obtained in our study.(18) According to Mueller et al., the highest diagnostic accuracy in NT-ProBNP, pg/ cc 825 was reported with $82 \%$ sensitivity and $81 \%$ specificity, while the sensitivity and specificity in our study were 95\% and $89 \%$, respectively. This was slightly different compared to Mueller. This difference can be attributed to the age range of the subjects under study, the frequency of sex and the systolic intensity of left ventricular dysfunction.(4)

The fQRS is a readily available, inexpensive and noninvasive ECG parameter. Cardiac remodelling can produce scar or fibrotic tissue, causing congestive heart failure, hemodynamically significant arrhythmias and cardiovascular death. The presence of fQRS can predict myocardial perfusion and left ventricular function. In addition, the results showed that narrowed fQRS could increase the incidence of major adverse cardiovascular complications, including hemodynamically significant ventricular arrhythmic complications, hypertrophic obstructive cardiomyopathy, acute coronary syndromes, decompensated systolic heart failure and non-ischemic cardiomyopathy. $(19,20)$ FQRS on ECG can serve as be used to detect myocardial fibrosis. $9.2 \%$ of healthy middle-aged Finnish subjects were reportedly received fQRS. However, higher prevalence of fQRS were observed in several diseases, including cardiac sarcoidosis (75\%), ST elevation myocardial infarction (21.9\%) and chronic kidney disease (60\%).(21)

\section{CONCLUSIONS}

FQRS is a widely used method for HF patients. The results showed a significant relationship between fQRS and the higher levels of NT-proBNP. The fQRS was associated with increased NT-proBNP and pronounced LV end-diastolic pressure in heart failure patients. 


\section{REFERENCES}

[1] Tanai E, Frantz S. Pathophysiology of heart failure. Compr Physiol 2015;6(1):187-214.

[2] Kurmani S, Squire I. Acute heart failure: definition, classification and epidemiology. Curr Heart Fail Rep 2017;14(5):385-92.

[3] Dharmarajan K, Rich MW. Epidemiology, pathophysiology and prognosis of heart failure in older adults. Heart Fail Clin 2017;13(3):417-26.

[4] Mueller T, Gegenhuber A, Poelz W, et al. Diagnostic accuracy of $B$ type natriuretic peptide and amino terminal proBNP in the emergency diagnosis of heart failure. Heart 2005;91(5):606-12.

[5] Libby P, Bonow RO, Mann DL, et al. Braunwald's Heart Disease: a textbook of cardiovascular medicine. Vol. 2. Elsevier Health Sciences Nov 21, 2007.

[6] Chen AA, Wood MJ, Krauser DG, et al. NT-proBNP levels, echocardiographic findings, and outcomes in breathless patients: results from the ProBNP Investigation of Dyspnoea in the Emergency Department (PRIDE) echocardiographic substudy. European Heart Journal 2006;27(7):839-45.

[7] Maisel AS, Krishnaswamy P, Nowak RM, et al. Rapid measurement of B-type natriuretic peptide in the emergency diagnosis of heart failure. New England Journal of Medicine 2002;347(3):161-7.

[8] Januzzi Jr JL, Chen-Tournoux AA, Moe G. Amino-terminal pro-B-type natriuretic peptide testing for the diagnosis or exclusion of heart failure in patients with acute symptoms. The American Journal of Cardiology 2008;101(3):S29-38.

[9] Ozcan F, Turak O, Canpolat U, et al. Myocardial tissue perfusion predicts the evolution of fragmented QRS in patients with ST-segment elevation myocardial infarction undergoing primary percutaneous coronary intervention. Ann Noninvasive Electrocardiol 2014;19(5):454-61.

[10] Keeley EC, Boura JA, Grines CL. Primary angioplasty versus intravenous thrombolytic therapy for acute myocardial infarction: a quantitative review of 23 randomised trials. Lancet 2003;361(9351):13-20.
[11] Azevedo JC, Reis BCC, Barreto NMPB, et al. BNP was associated with ischemic myocardial scintigraphy and death in patients at chest pain unit. Arq Bras Cardiol 2015;104(1):16-23.

[12] Onoue Y, Izumiya Y, Hanatani S, et al. Fragmented QRS complex is a diagnostic tool in patients with left ventricular diastolic dysfunction. Heart Vessels 2016;31(4):563-7.

[13] Al-Habeeb W, Al-Ayoubi F, Al-Ghalayini K, et al. Saudi Heart Association (SHA) guidelines for the management of heart failure. J Saudi Heart Assoc 2019;31(4):204-53.

[14] Sheng QH, Hsu CC, Li JP, et al. Combining fragmented QRS and TIMI score for predicting in-hospital short-term prognosis after acute myocardial infarction. J Zhejiang Univ Sci B 2018;19(5):349-53.

[15] Richards AM. N-terminal b-type natriuretic peptide in heart failure. Heart Fail Clin 2018;14(1):27-39.

[16] Zhang R, Chen S, Zhao Q, et al. Fragmented QRS complex is a prognostic marker of microvascular reperfusion and changes in LV function occur in patients with ST elevation myocardial infarction who underwent primary percutaneous coronary intervention. Experimental and Therapeutic Medicine 2017;13(6):3231-8.

[17] Barman HA, Durmaz E, Atici A, et al. The relationship between galectin-3 levels and fragmented QRS (fQRS) in patients with heart failure with reduced left ventricular ejection fraction. Ann Noninvasive Electrocardiol 2019;2:e12671.

[18] Zhao Q, Zhang R, Hou J, et al. Relationship between fragmented QRS and NT-proBNP in patients with ST elevation myocardial infarction who underwent primary percutaneous coronary intervention. Acta Cardiologica Sinica 2018;34(1):13-22.

[19] Das MK, Saha C, El Masry H, et al. Fragmented QRS on a 12-lead ECG: a predictor of mortality and cardiac events in patients with coronary artery disease. Heart Rhythm 2007;4(11):1385-92.

[20] Maisel AS, Duran JM, Wettersten N. Natriuretic peptides in heart failure: atrial and b-type natriuretic peptides. Heart Fail Clin 2018;14(1):13-25.

[21] Sadeghi R, Dabbagh VR, Tayyebi M, et al. Diagnostic value of fragmented QRS complex in myocardial scar detection: systematic review and meta-analysis of the literature. Kardiol Pol 2016;74(4):331-7 